\title{
Multifidus Muscle Volume Estimation Based on Three Dimensional Wavelet Multi Resolution Analysis: MRA with Buttocks Computer- Tomography: CT Images
}

\author{
Kohei Arai ${ }^{1}$ \\ Graduate School of Science and Engineering \\ Saga University \\ Saga City, Japan
}

\begin{abstract}
Multi-Resolution Analysis:. MRA based edge detection algorithm is proposed for estimation of volume of multifidus muscle in the Computer Tomography: CT scanned image The volume of multifidus muscle would be a good measure for metabolic syndrome rather than internal fat from a point of view from processing complexity. The proposed measure shows 0.178 of $R$ square which corresponds to mutual correlation between internal fat and the volume of multifidus muscle. It is also fund that $\mathbf{R}$ square between internal fat and the other possible measures shows smaller than that of multifidus muscle.
\end{abstract}

\section{Keywords—Edge detection; MRA; Multifidus muscle}

\section{INTRODUCTION}

A measure for representing metabolic syndrome based on Computer Tomography: CT value of multifidus is proposed by Professor Eguchi of Saga University. It is still difficult to treat CT value for expressing levels of metabolic syndrome. The proposed method allows representation of volume of multifidus easily by using discrete wavelet transformation. I have calculated the ratio of the subcutaneous fat $\mathrm{CT}$ value and this intra-abdominal fat volume, multifidus CT value / fat CT value ratio, the waist muscle $\mathrm{CT}$ value / fat $\mathrm{CT}$ value ratio. As a result, I asked that the subcutaneous fat $\mathrm{CT}$ value ratio of multifidus waist muscle correlates with muscle fatty.

Iinterspinous muscle, the transversospinalis muscle, etc. , multifidus of transversospinalis muscle especially susceptible to stress, it is effective in preventing the pain that occurs by stimulating the peripheral nerves of the fascia and hyperemia in psoas muscle, swelling, inflammation and. Further, to train the multifidus leads to enhancing the motor functions of the spinal column as a whole. Further, it is possible volume of multifidus can be used as an index of abdominal fat high correlation with it, to give an indication of when recovering from metabolic syndrome based on this indication. Volume of the multifidus muscle can be estimated from X-ray CT images. Further, it is also possible to obtain from 3D Discrete Cosine Transformation: DCT image directly the volume of intraabdominal fat, the time and more effort is applied to this,
It is not possible to achieve practical processing time. Further, important method of estimating the multifidus volume by reducing the artifacts from the image obtained with a small dose in this analysis [1]-V[7]. Contour extraction method using a differential operator conventionally has been widely used for this analysis. The contours extracted by the differential operator, there are drawbacks such as low in general, the contour does not (1) intended to be extracted is more, resistance to (2) noise. Since the extracted contour by differential operator would extract the component regarded as a contour in the entire frequency range, contour unintended and would be extracted, for extracting a contour high frequency components of the noise uniformly distributed in frequency, noise immunity is low.

Determining the intra-abdominal fat volume from the abdominal CT images using the "Fat Scan." is proposed Furthermore, to examine the relationship between intraabdominal fat to obtain a $\mathrm{CT}$ value of multifidus and buttocks volume. In order to determine the relationship between the multifidus and abdominal fat, the method is performed with (Multi Resolution Analysis: MRA) 3D wavelet multiresolution analysis and two-dimensional MRA. In order to avoid this, this paper deals with wavelet multi-resolution analysis: propose contour extraction with MRA [8],[9]. Since MRA is equivalent to the filter bank, it is possible to extract the contour of a desired frequency component. I seek the MRA up to four levels in each dimension. I determine the value of intra-abdominal fat and multifidus number of multifidus at each stage. Furthermore, I ask each of the value of intra-abdominal fat and multifidus number of multifidus performs morphological analysis after performing each MRA. The following section describes the research background and the proposed method followed by some experimental data analysis with acquired CT images of patients. Then conclusion is described together with some discussions.

\section{PROPOSED Method}

\section{A. Moltifidus}

Multifidus is a muscle that runs on inner side of the upper vertebra of "spinous process" between the "horizontal projection" and transversospinalis muscle is. I divided into 


\section{(1) semispinalis, \\ (2) multifidus, to \\ (3) rotator}

and further divided the transversospinalis muscle. Semispinalis spread to vertebrae of five to six. Multifidus is located deeper than the semispinalis muscle spread vertebrae three or four, as shown in Figure 1.

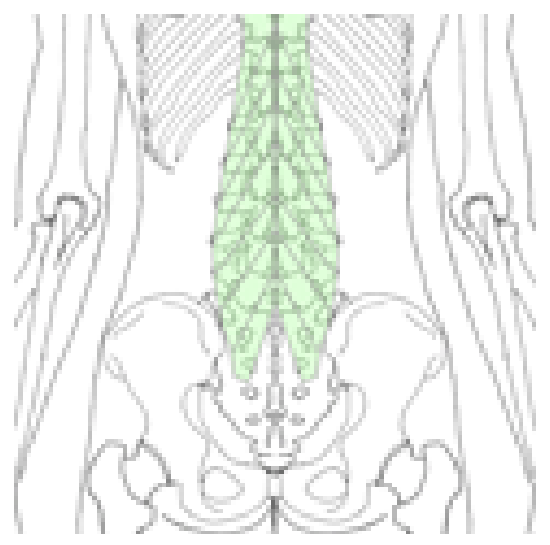

Fig. 1. Multifidus muscle

Furthermore, rotator spread between two vertebrae from one, is located in the deepest. With the contraction of the transversus abdominis, multifidus is activated; pull the spinous process, to rotate to the other side. There is a rotation movement turn around the back, such as turning the waist. (Slope, the latter is tilted backward sacral sacrum before the former) to control the position of the sacrum, the transverse abdominal muscle contraction increases the tension of the back muscles chest film outside, iliococcygeus muscle- coccyx muscle and multifidus to increase intra-abdominal pressure to stabilize the lumbar spine by you. Furthermore, by using in conjunction with the outer unit, and increase the tension of the rear sacroiliac ligament through the chest spine film, transversus abdominis contributes to closing strengthen pelvic girdle. Load of 12 pieces of work in each facet. Is a translation of the six rotatory motion of six around XYZ axes and (Mo), along the axes. (Spinal muscle, the longest streak, iliocostalis muscle (rotator muscles, semispinalis, multifidus) erector spinae transversospinalis muscle called the intrinsic muscles of the back's move freely at will exercise the 12 interspinous muscle, is a intertransversalis,). Muscle short mileage as located in the deep, to be involved in the finer movement.

\section{B. Edge extraction and buttocks CT image}

Figure 2 shows an example of edge extraction Laplacian operator hip and CT images. As shown in Figure 2, the edge of the desired other than, well, there is a tendency for noise as well as to emphasize edge extracted Sobel, the Laplacian operator or the like.

\section{Wavelet Multi Resolution Analysis: MRA}

2D and 3D wavelet transformation is illustrated in Figure 3 (a) and (b), respectively. Original image can be divided into four components $\mathrm{LL}_{1}, \mathrm{LH}_{1}, \mathrm{HL}_{1}$, and $\mathrm{HH}_{1}$ where $\mathrm{L}$ and $\mathrm{H}$ denote Low frequency and High frequency components and the order of $\mathrm{L}$ and $\mathrm{H}$ is Horizontal and Vertical directions (Pixel and Line directions).
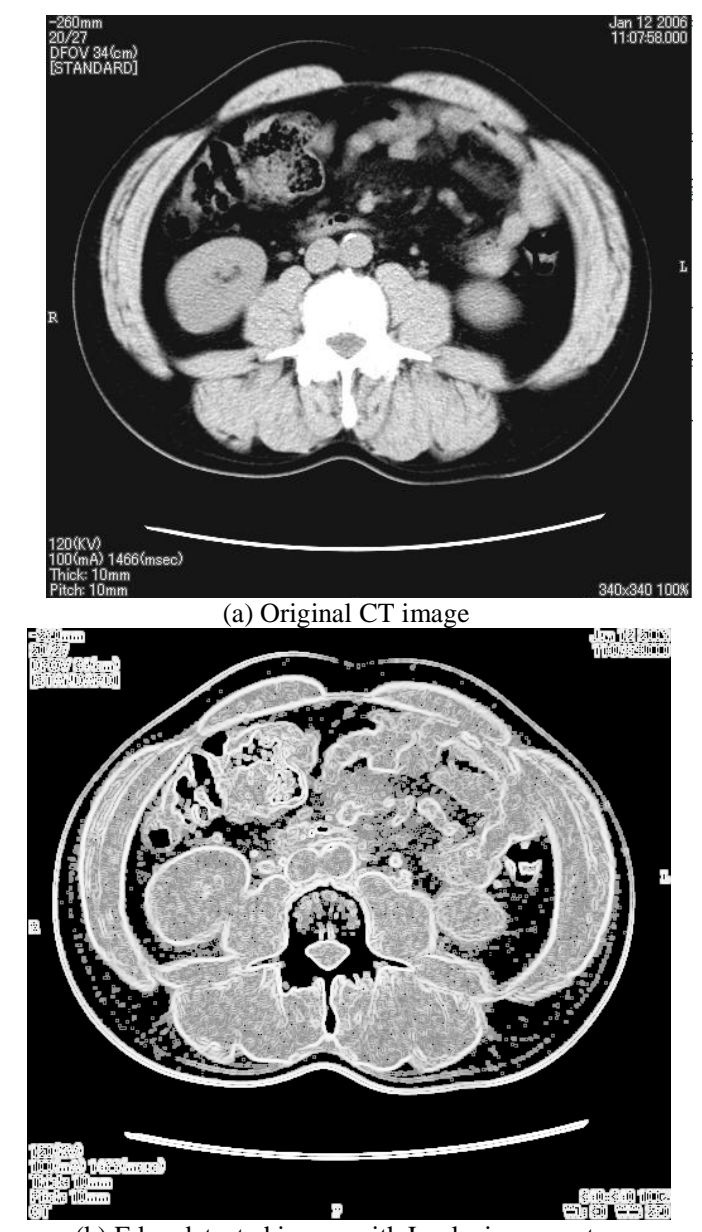

(b) Edge detected image with Laplacian operator

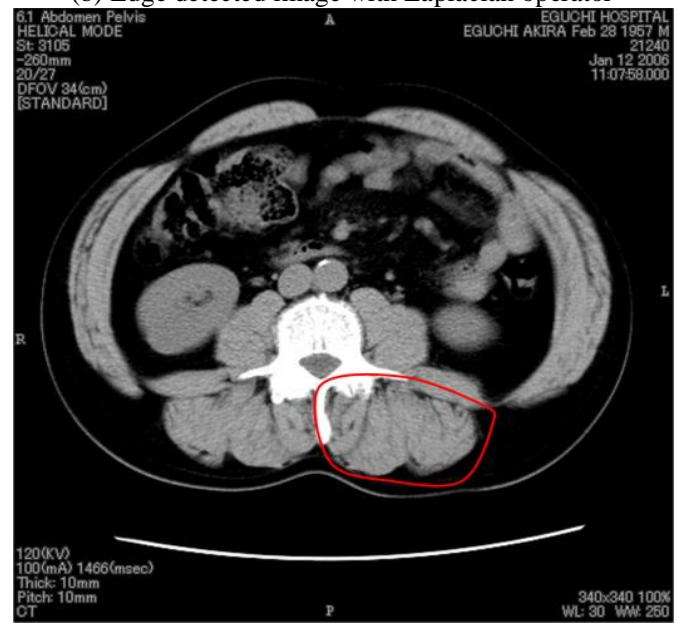

(c)Mu8ltifidus Lo9cation (red lines shows)

Fig. 2. Original image and edge detected image with Laplacian operator.

On the other hand, suffix denotes level. $\mathrm{LL}_{1}$ component can be divided into $\mathrm{LL}_{2}, \mathrm{LH}_{2}, \mathrm{HL}_{2}$, and $\mathrm{HH}_{2}$. Furthermore, $\mathrm{LL}_{2}$ can also be divided into $\mathrm{LL}_{3}, \mathrm{LH}_{3}, \mathrm{HL}_{3}$, and $\mathrm{HH}_{3}$. Generally, $\mathrm{LL}_{\mathrm{n}}$ can be divided into $\mathrm{LL}_{\mathrm{n}+1}, \mathrm{LH}_{\mathrm{n}+1}, \mathrm{HL}_{\mathrm{n}+1}$, and $\mathrm{HH}_{\mathrm{n}+1}$. Importantly, original image can be reconstructed with all these components perfectly.

Meanwhile, an example of edge detected image based on 2D Multi-Resolution Analysis: MRA is shown in Figure 3(c). 


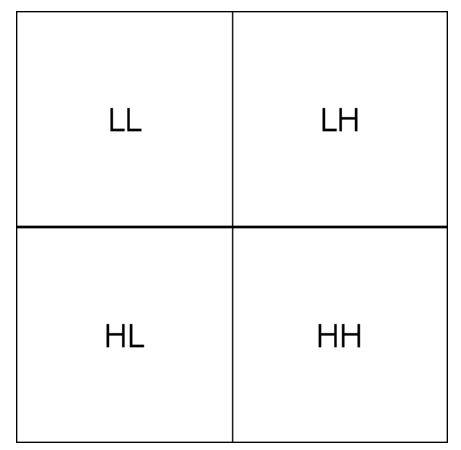

(a) $2 \mathrm{D}$ wavelet transformation

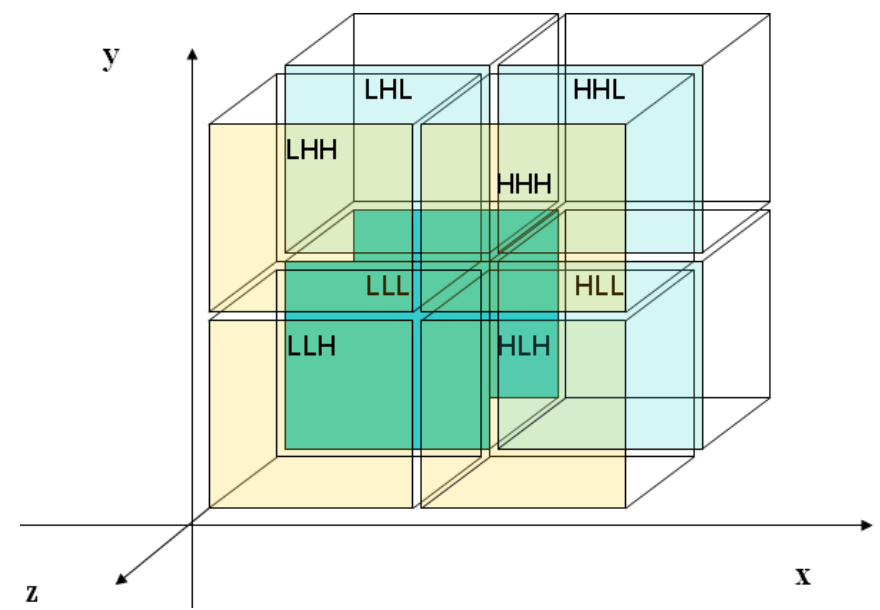

(b) 3D wavelet transformation

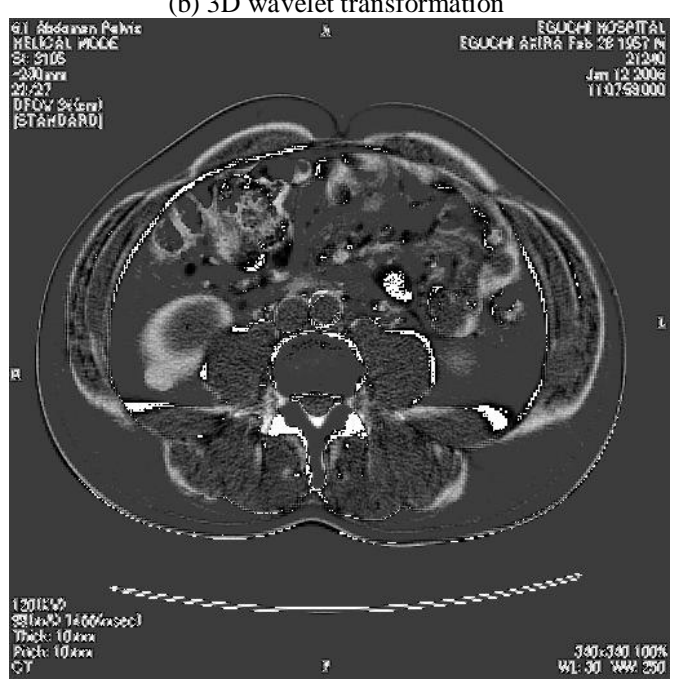

(c) An example of edge detected image based on 2D Multi-Resolution Analysis: MRA

Fig. 3. 2D and 3D wavelet transformation and an example of edge detected image based on 2D MRA

Multifidus in concern is composed with a number of ellipsoidal shapes of muscles. Therefore, string types of edges are seen at the multifidus. These edges are detected by $2 \mathrm{D}$ and 3D MRA. Edge is essentially high frequency components. Therefore, edges are detected by reconstructing image using $\mathrm{LH}$, or $\mathrm{HL}$, or $\mathrm{HH}$ components after MRA. If reconstruction is made with LH component only, then horizontal edges can be detected while vertical edges can be detected by reconstructing image using HL component only. Both horizontal and vertical edges can also be detected by reconstructing using $\mathrm{HH}$ component only. Some other combinations among LH, HL, $\mathrm{HH}$ are also available and useful in some cases.

\section{EXPERIMENGTS}

\section{A. Evaluation function}

It has been proposed can be an indicator of the function of the multifidus the size of the multifidus indicators proposed, it is intended that the risk of metabolic syndrome and the like and to provide a measure of the recovery degree from this. Therefore, the index is necessary that correlation between the abdominal fat is high. Body Mass Index: BMI, etc. (the ratio of the CT values of visceral fat and multifidus) fat volume, subcutaneous fat volume, waist muscle volume, multifidus volume, CT value ratio is proposed as an indicator whole:

Body Mass Index: BMI as an indicator of the traditional are. R-square value of the intra-abdominal fat is as high as among these indicators, and selects the one that can be extracted relatively easily from hip CT images. It is obtained by dividing the square of the height of the body weight, BMI can find out very easily. Obtained from buttock CT image but otherwise, CT value of the ratio can be calculated relatively easily identify the pixels if possible. Psoas volume, multifidus volume can be calculated relatively easily since only factor of the pixel if you can even contour extraction.

Specific fat cells difficulties subcutaneous fat, whole fat as well as intra-abdominal fat. Thus, by comparing the calculation result of the multifidus volume based MRA is proposed indices as correct the result of the stochastic by expert judgment indicators conventionally. These were of the proposed indicator in this paper. Judgment result of experts determined from the CT images for subjects of 362 names, up to 81 years from 25 years in Figure 4, ie, shows the relationship of the conventional index and intra-abdominal fat as a correct answer. For each of the posies muscle volume fat volume, subcutaneous fat volume, CT value ratio, and multifidus whole volume, it has become $0.408,0.045,0.001$, 0.178 , and 0.111 , R-square values, correlation with overall fat volume I know that but the highest. However, since the time and effort-consuming in order to determine the volume as described above, multifidus volume obtained processing time in less relatively high $\mathrm{R}$-square value is then this judgment indicative of suboptimal was Level and 3.2.

\section{B. Example of wavelet transformation}

Figure 4 shows examples of the original CT image, and 2D as well as 3D wavelet transformed images. Obviously, 3D wavelet transformed image has much edge related information rather than $2 \mathrm{D}$ wavelet.

\section{Tested People}

The following four patients are participated to the experiments,

$$
\text { Age: } \quad 72,63,59,41
$$

Height $(\mathrm{cm})$ : Weight $(\mathrm{kg})$

164:71, 170:75, 160:76, 156:62

Body Mass Index: BMI: 26.21, 25.95, 29.69, 24.22 

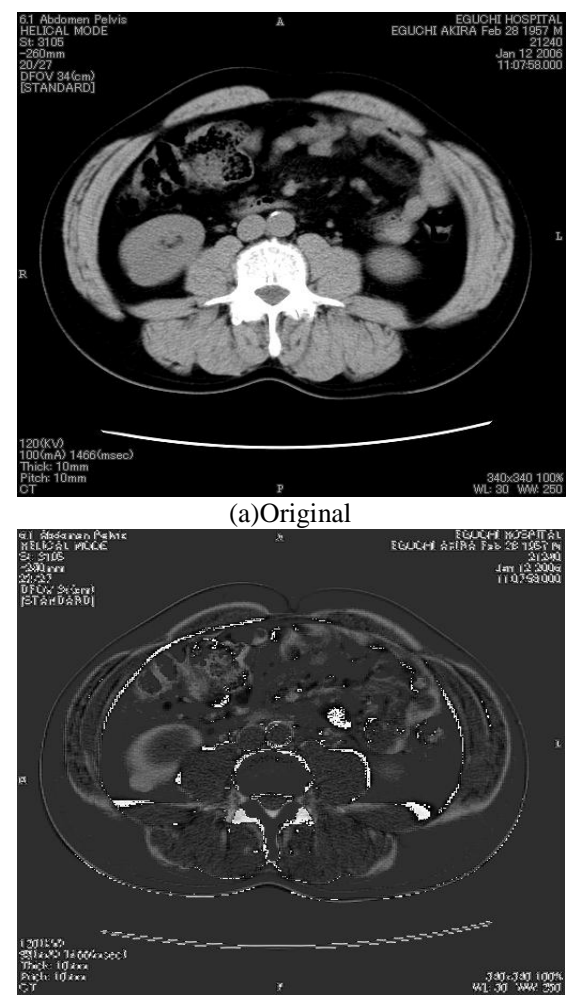

(b)2D wavfelet transformation (level 1)

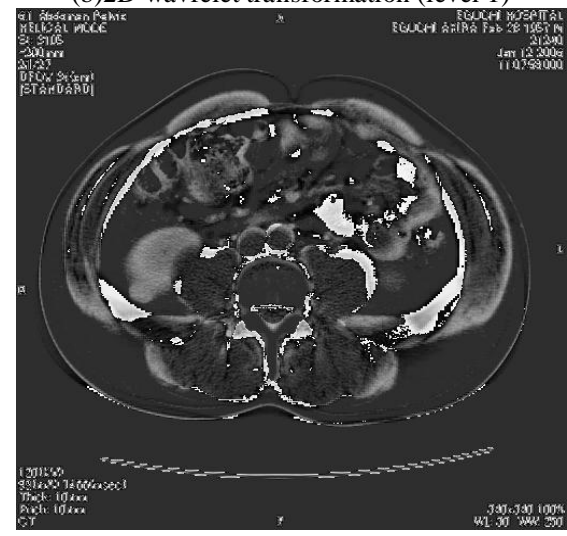

(c)3D wavelet transformation

Fig. 4. Examples of wavelet t5ransfo0rmed images

\section{Process flow}

In order to extract much clear edge information, pixel labeling followed by morphological filtering (dilation and erosion) is applied to the wavelet transformed images. Figure 5 shows illustrative view of pixel labeling. Meanwhile, Figure 6 shows morphological filtering.
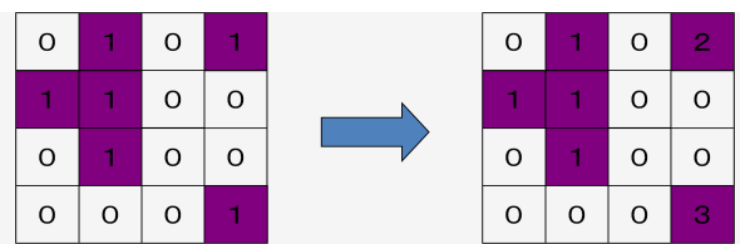

Fig. 5. Pixel labeling

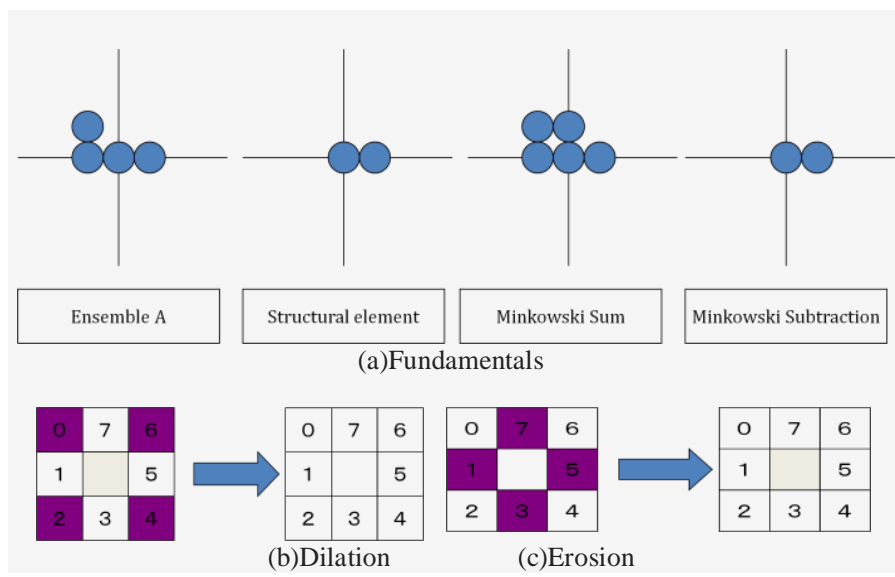

Fig. 6. Morphological filtering

\section{E. MRA basis function}

Using the Daubechies and Haar as a basis function, is subjected to MRA for the buttocks CT image by changing to level $1,2,3$, and 4 , we attempted to outline extraction of multifidus. It is shown in Figure 5 an example of the result. (a), (b), (c) is a rump CT original image at different sites, respectively. (d), (e), (f) is a contour image reconstructed except for the LL component 2DMRA at two levels. (g), (h), (i) is a contour image reconstructed with the exception of the LLL component of 3DMRA in level 2. It is as 1 Table to extract the contour from the contour image that is obtained in this manner, to examine the $\mathrm{R}$-square value of the intraabdominal fat volume by calculating the (number of pixels ie) multifidus volume was found. That is, the difference between $\mathrm{R}$-square value by the base functions is hardly observed, levels for specifying the frequency contour is found that 2 or, 3 is good.

TABLE I. R-SQUARE VALUE BETWEEN STOMACH FAT AND VOLUME OF MULTIFIDUS MUSCLE ESTIMATED THROUGH MRA WITH 1-4 LEVELS

\begin{tabular}{|l|l|l|l|l|}
\hline Level & 1 & 2 & 3 & 4 \\
\hline Haar & 0.160 & 0.175 & 0.177 & 0.169 \\
\hline Duabechies & 0.162 & 0.178 & 0.178 & 0.172 \\
\hline
\end{tabular}

\section{F. Experimental Results}

Figure 7 shows examples of edge detected images through reconstruction without LL component (2D MRA) and LLL (3D MRA) from 1 to 4 levels of the wavelet transformed images.(a), (b) and (c) are original CT images at the different height (portion). (d), (e) and (f) are edge detected images reconstructed without LL component. (g), (h) and (i) are edge detected images reconstructed without LLL component.

Meanwhile, Figure 8 shows relations between stomach fat and the other measured factors. 

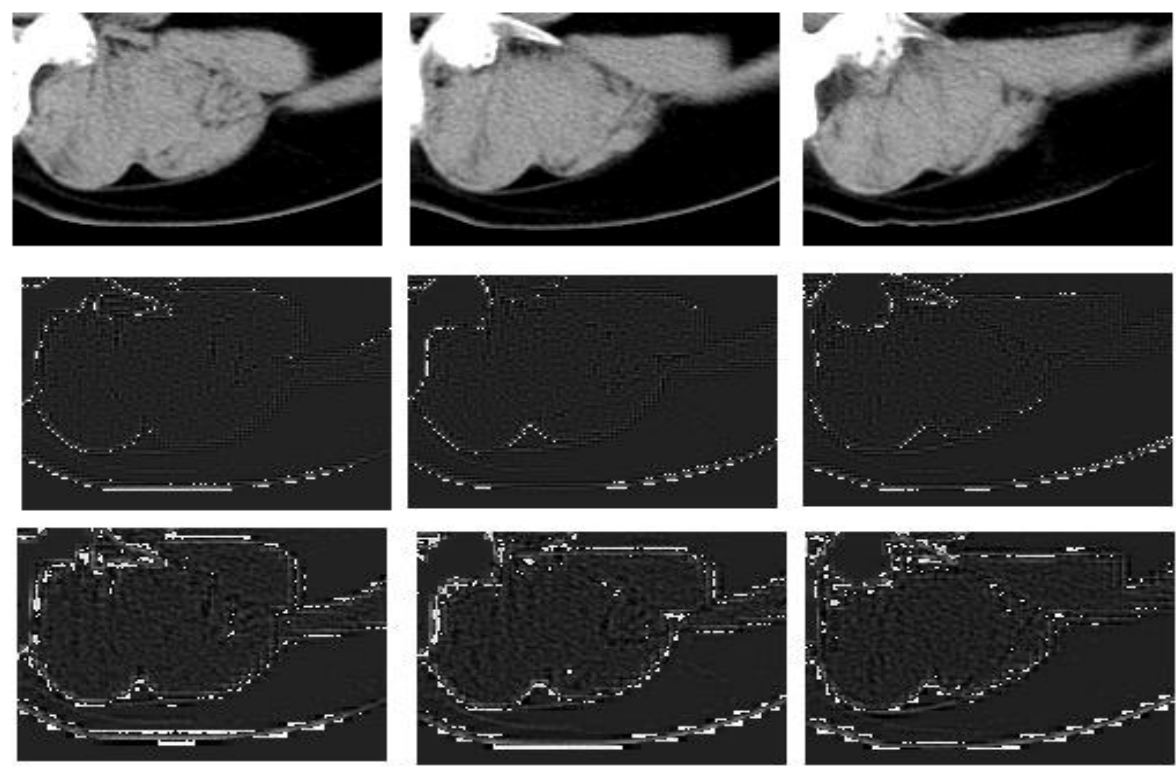

(a)2D wavelet transformed images
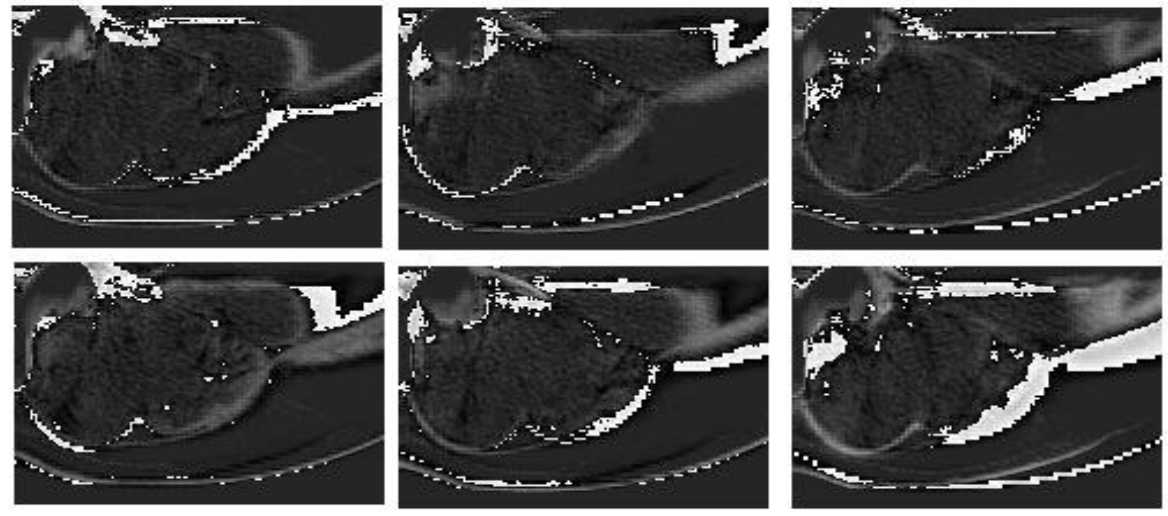

(b)3D wavelet transformed images
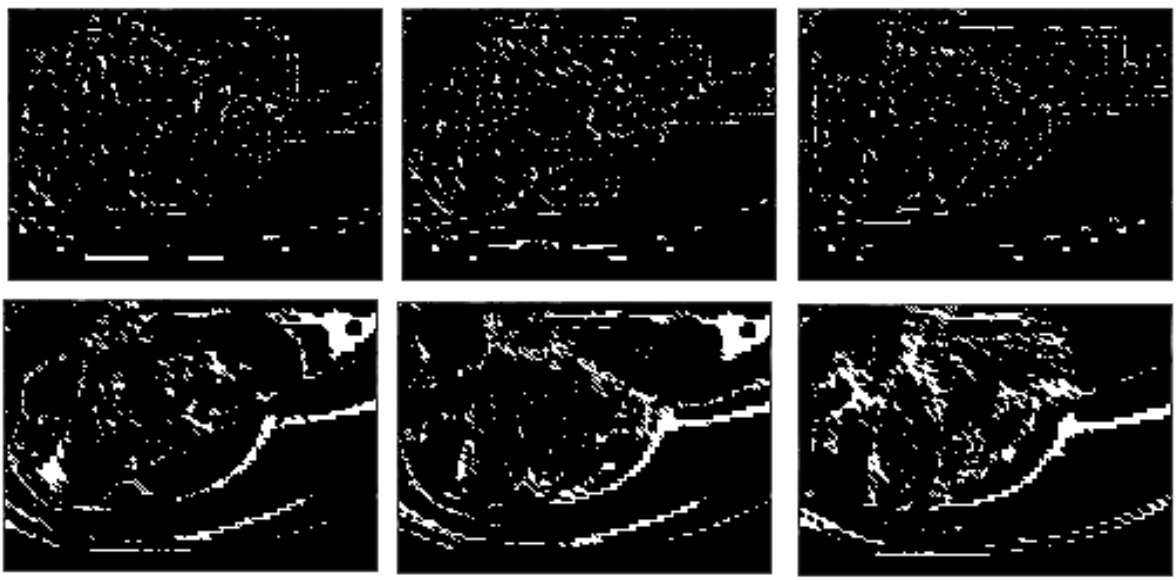

(c)After morphological filtering

Fig. 7. Examples of edge detected images through reconstruction without LL component (2D MRA) and LLL (3D MRA) from 1 to 4 levels of the wavelet transformed images.(a), (b) and (c) are original CT images at the different height (portion). (d), (e) and (f) are edge detected images reconstructed without LL component. (g), (h) and (i) are edge detected images reconstructed without LLL component. 


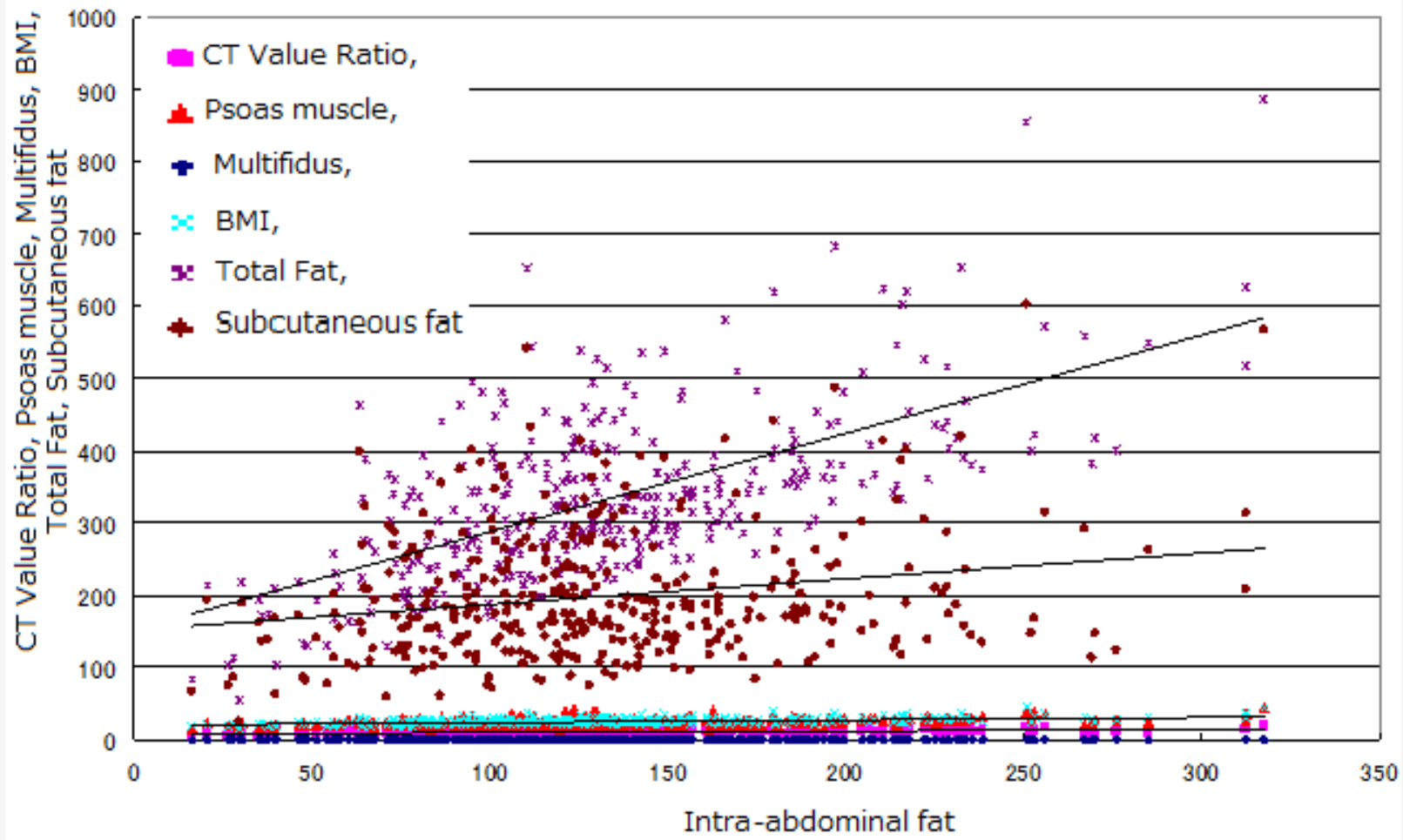

Fig. 8. Relations between stomach fat and the other measured factors

For intra-abdominal fat and multifidus volume, an increase of intra-abdominal fat is observed volume of the multifidus muscle is reduced. It is also found that multifidus adipose CT value ratio increases with increasing of abdominal fat.

If you look at the value of intra-abdominal fat and number of multifidus on the basis of the value of intra-abdominal fat and CT value ratio, these are not seen feature in both 2D DWT, 3D DWT of each stage when you do not subjected to morphological analysis. It is believed that unchanged edges of multifidus are observed in a certain value in order to present numerous. However, if you have applied twice morphological analysis, for the closing and closing, 3D DWT case shows much better relation between intra-abdominal fat and multifidus volume than the 2DW cases.

In particular, it can be obtained a high value by performing a two-step MRA of 3D DWT, is applied twice closing. Furthermore, since it is the result of as if they are subjected twice when closing subjected three times more than the closing, closing it is found that it may be subjected to two times.

Although not observed in the characteristic value if 2 times erosion together with dilation. This is presumably because the edges of the multifidus should not lead. By performing two times until dilation are captured

\section{CONCLUSION}

It is found that multifidus volume of intra-abdominal fat can be estimated with CT image through 3D wavelet transformation much clearly than 2D wavelet transformation. Traditionally, although intra-abdominal fat can be estimated with $\mathrm{CT}$ value, it is found that the relation between $\mathrm{CT}$ value and intra-abdominal fat is not so high. Meanwhile, relation between intra-abdominal fat and total fat is obviously high followed by subcutaneous fat, multifidus, BMI, psoas muscle, and CT value ratio. Therefore, multifidus volume can be an indicator of intra-abdominal fat. Because of 3D wavelet MRA allows estimation of multifidus volume, it is useful for measure intra-abdominal fat with CT image derived multifidus volume.

\section{ACKNOWLEDGMENT}

The author would like to express a sincere gratitude to Professor Dr. Eguchi Yuichiro of the Faculty of Medicine in Saga University for his courtesy the data subject at the beginning of this study. Furthermore, author also would like to thank Mr. Mitsuo Mochida for his cooperation through experiment.

\section{REFERENCES}

[1] CT Performance Evaluation Committee : Standard for Performance Evaluation of X-ray computer tomography apparatus ( second recommendation ), the Japan Medical Association Journal , 88 ( 8 ),759 $-771,1998$.

[2] Technical Committee X-ray CT system performance evaluation study group : Standard for X-ray CT system performance evaluation (draft ), Nippo technology magazine Science, 47 ( 1 ) ,56 -63 , (1991)

[3] Suzuki Kenji other technical problem of helical CT system, Nippo skill science magazine, 52 ( 3 ) 384 -388 (1996)

[4] TSUJIOKA Katsumi : Nippo skill science magazine, operation - and the actual technical problems - performance evaluation of helical CT system, 52 ( 3 ) ,389 -396 (1996)

[5] Muramatsu, Tadashi Hisashi other basic performance of helical scan CT, Nippo skill science magazine, 52 ( 1 ),81 -85 (1996) 
[6] Keiko Kubota other applications of helical scan in the base of the brain, Nippo skill science magazine , 52 ( 9), $1066,(1996)$

[7] other Shun Kuwahara Sada : performance evaluation of HiSpeed AdvantageRp, ,56,51-58 (1996) technology magazine -release north

[8] Kohei Arai : basic theory of wavelet analysis , Morikita publication (2000)

[9] Kohei Arai : self-study wavelet analysis , modern science, Inc. ( 2006 )

\section{AUTHORS PROFILE}

Kohei Arai, He received BS, MS and PhD degrees in 1972, 1974 and 1982, respectively. He was with The Institute for Industrial Science and Technology of the University of Tokyo from April 1974 to December 1978 also was with National Space Development Agency of Japan from January, 1979 to March, 1990. During from 1985 to 1987, he was with Canada Centre for Remote Sensing as a Post Doctoral Fellow of National Science and Engineering Research Council of Canada. He moved to Saga University as a Professor in Department of Information Science on April 1990. He was a councilor for the Aeronautics and Space related to the Technology Committee of the Ministry of Science and Technology during from 1998 to 2000 . He was a councilor of Saga University for 2002 and 2003. He also was an executive councilor for the Remote Sensing Society of Japan for 2003 to 2005. He is an Adjunct Professor of University of Arizona, USA since 1998. He also is Vice Chairman of the Commission "A" of ICSU/COSPAR since 2008. He wrote 30 books and published 472 journal papers 